SARS

\title{
SARS: experience from the emergency department, Tan Tock Seng Hospital, Singapore
}

\section{E Seow}

\section{Lessons learnt}

$\mathrm{T}$ he severe acute respiratory syndrome (SARS) entered Singapore through three young women, who were in Hong Kong from 20 to 24 February 2003. ${ }^{1}$ They were infected by a doctor from Guangzhou by a chance encounter in the lift lobby of the hotel where all were staying. ${ }^{2}$

Two were admitted to Tan Tock Seng Hospital (TTSH) and one of them was what the World Heath Organisation (WHO) later described as a "superspreader" (persons who directly infected $\geqslant 10$ other persons $\left.{ }^{3}\right)$.

On the 22 March 2003, the Singapore government made a decision to centralise the care of suspect and probable cases of SARS including paediatric cases in TTSH. This facilitated the management of SARS patients and reduced the risk of secondary transmission of the disease. ${ }^{4}$ Ambulances were diverted and patients at the emergency department (ED) with non-SARS conditions who required hospitalisation were transferred to other hospitals.

The screening centre was initially at the Centre for Communicable Disease (within the TTSH campus). This moved to the ED on the 26 March 2003. The ED then became the national screening centre.

By the 31 May 2003 when WHO took Singapore off the list of SARS affected countries, we had screened more than 9000 patients.

The aim of this article is to describe our ED experiences in dealing with this outbreak and the lessons learnt.

\section{INITIAL WARNING -THE FIRST WEEK (14 MARCH-20 MARCH 2003)}

Retrospectively, the first warning we received was through our emails on the 13 March 2003. The Ministry of Health, Singapore had sent out a medical alert warning of an outbreak of atypical pneumonia in Hong Kong, Vietnam, and Guangdong province in China. The medical alert commented that there were three cases that had returned from Hong Kong who had been treated for pneumonia but were well. Two had been discharged and one was recovering. It also stated that none of the hospital staff attending to these patients had reported ill but advised hospital staff attending to this type of cases to take the necessary infection control measures.

Our response in the ED was to obtain a travel history from febrile patients and give a surgical facemask to at risk patients. There was no sense of danger until the next evening when one of our infectious disease (ID) physicians came to ED to warn us of this unknown entity. It was only then that a decision was made by the ED consultant on duty and the head of ED to physically separate at risk patients from the other patients. They were placed in our decontamination building, which was adjacent to the ED (area D in figure 1). The at risk patients were attended to in area D by staff wearing PPE (personal protection equipment), which consisted of gloves, gown, and a N95 mask. We would add goggles or a visor to this armamentarium a month later.

Our triage nurses started to don N95 masks at this time as well.

During this visit, the ID physician also informed us that there was a group of ward staff that had been admitted for fever. The ED therefore began categorising TTSH personnel as at risk despite the absence of a formal announcement. As a result a member of staff waiting to be admitted in our observation room was transferred to area D to wait for a bed and another, seen earlier in the day and discharged, was recalled for admission.

TTSH opened its emergency operations room 24 hours a day from the 15 March 2003, day 2 of the crisis. Daily meetings to plan and manage the crisis were chaired by the chief executive officer of TTSH and attended by heads of various areas including the head of ED.

I began to write open letters to my staff on an almost daily basis to keep them updated. These were issued to all grades of staff.

On day 3, all ED staff wore N95 facemasks regardless of work area. We were still managing our regular load of about 380 patients a day (TTSH ED was the busiest ED in Singapore) and were depending on our triage nurses to sieve out at risk patients. Any patients who slipped through triage would be waiting in the same area as other patients.

It was only on day 4 ( 17 March 2003) that a portable radiograph machine was placed in area D for the dedicated use of at risk patients. Before this, the at risk patients had their chest radiograph taken in the ED in the same area as other patients.

The next day, a screening station was set up outside the department to separate at risk cases from "other patients". The latter were then triaged inside the ED as per normal practice. It was at the same time that an ED staff presented with fever. Eventually, 10 more would be admitted for observation.

By day 6, one of our epidemiologists began to inform us directly of at risk areas. This enabled us to carry out further revisions of our screening questionnaire in a timely fashion and which by then was into its third draft.

With respect to the hospital, the intensive care units were closed to non-SARS patients but ED was still receiving ambulance cases.

\section{THE SECOND WEEK (2 1 MARCH-} 27 MARCH 2003)

By the eighth day all ED staff involved in patient care were in full PPE. We were still operating with our usual load as well as screening a small number of patients.

On 22 March 2003, the Ministry of Health designated TTSH as the SARS hospital. It meant that "we were to stop ED admissions, stop admissions/transfers from other hospitals unless the patients were SARS related. The nonSARS patients still in the hospital, we were to gradually discharge them when they were better, and so empty our wards to only look after SARS related patients. The outpatient clinics were also closed." ${ }^{\prime 6}$

ED was on ambulance diversion but continued to see walk in patients who were non-SARS.

Interestingly, the ED attendance did not decrease despite the fact we were declared the SARS hospital. A decision was made on the 23 March 2003 to

Abbreviations: PPE, personal protection equipment; ED, emergency department; ID, infectious disease; TTSH, Tan Tock Seng Hospital 


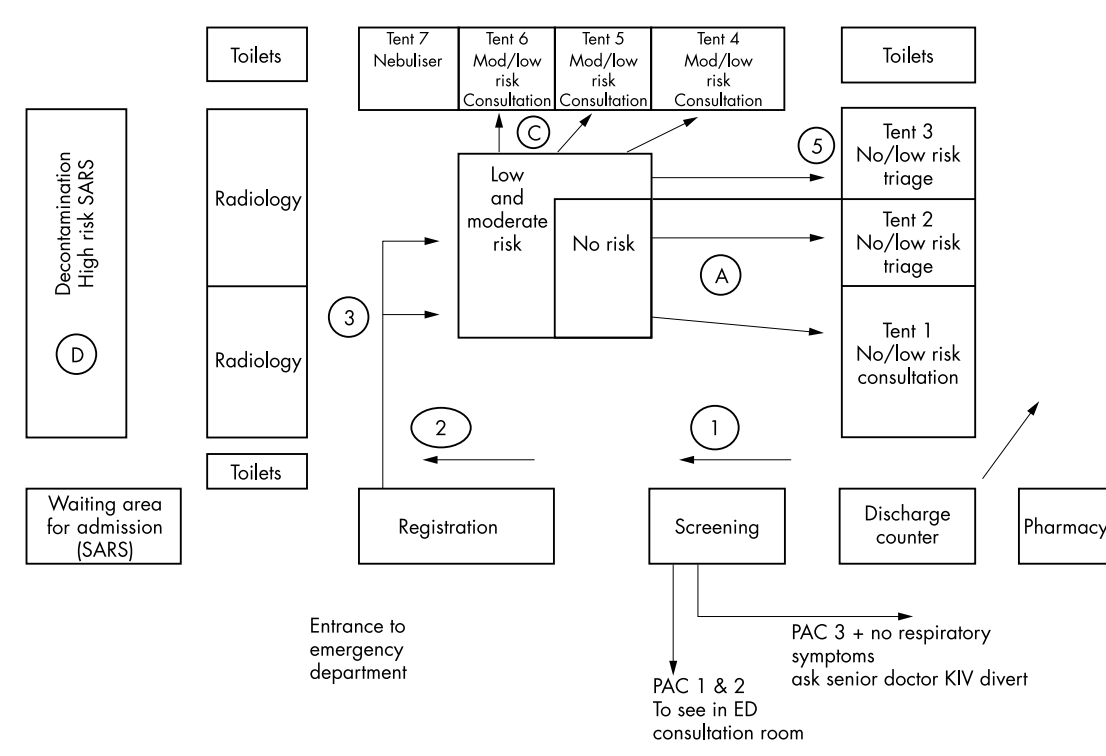

Figure 1 SARS screening and treatment area.

build tents under the car porch in front of the ED entrance (fig l). Those who had a temperature higher than $38^{\circ} \mathrm{C}$ were seen in area D and the others in the tents. The tents were operational by the next day (day 11). At the same time, two senior nurses were coopted to form the ED operational team to assist the head of department.

We revised the screening questionnaire again and began preparations to take over from CDC the screening of patients for SARS. We became the national screening centre on 26 March 2003 (day 13). A hotline was started with dedicated phone lines. It was run by vulnerable staff who we wanted to shield from direct patient contact (for example, those who were pregnant or had chronic illnesses). The hotline staff handled inquiries from patients ED had seen and was one of our safety nets. They worked closely with the Home Surveillance Group-our other safety net. This group would phone all the patients we had screened on the first three days after discharge and on alternate days thereafter until day 14. This had allowed us to recall and admit a significant minority for SARS that had not exhibited typical symptoms and signs in the first consultation.

The other responsibilities of the screening centre included keeping the Ministry of Health up to date about patients admitted because of SARS, liaison with officials of various agencies in charge of the country's borders and the police.

It was only on day 13 that ED was given the authority to actively turn away patients who had non-SARS related conditions unless they were critically ill. However, a safety mechanism was put in place-they had to be vetted by a senior doctor.
Staff were reminded to follow only instructions from the head of department.

\section{WEEK 3 (28 MARCH TO 3 APRIL 2003)}

Operations within the ED were moved outdoors to an enlarged tentage area. Administration of medications through the nebuliser route was done outdoors. Only patients who clearly did not have SARS, for example, dislocated shoulders were treated within the ED.

Standard letters were prepared such as fitness for travel, not having SARS, and discharge advisory. Revisions were made as new information was available.

A computerised database of at risk patients and their contacts was created by the information technology team of the organisation and was named the SARSweb. It took some time before this database stabilised.

When this was introduced, our screening nurses asked patients if they had any known contacts with SARS patients and if the answer was yes, the nurse would check the name against the database. However, after one patient did not tell the truth, the workflow changed. The screening nurses now actively checked the patient's name against the database. This added to the screening time.

The powered air positive respiratory hood was introduced for use in high risk activity like intubations but we did not use it when administering nebulisers until week 9.

\section{WEEK 4, 5 (4 APRIL TO 17 APRIL 2003)}

A new cluster of SARS patients from other hospitals emerged.
The names of persons accompanying patients and their contact numbers together with their temperatures were now logged in the screening questionnaire of every patient. This was to facilitate contact tracing.

Admissions from the ED was streamlined to only SARS or non-SARS. No categorisation of SARS patients at ED was permitted (this was an ED decision). It was difficult to categorise them at $\mathrm{ED}$ as the definitions given at that point were open to differing interpretations. In not permitting categorisation at ED, we ensured that all patients admitted for SARS or to exclude SARS were admitted to an isolation room for further review by the ID consultant.

Before the outbreak, TTSH ED had admitting rights. However, during the first four weeks of the SARS crisis, only ID physicians could admit to a SARS bed. On week 5, this authority was extended to the ED senior doctor. As a result, the turnaround time was short and streamlined the flow for these patients.

As a precautionary measure, ED admitted non-SARS patients with pneumonia or fever of indeterminate origin to isolation beds. The other group of non-SARS patients admitted to isolation beds were patients on home quarantine orders who were being admitted for non-SARS conditions. We were fortunate to have put this in place as a patient who was on home quarantine order, who was afebrile with no respiratory signs or symptoms on presentation and admitted because of anaemia subsequently manifested signs and symptoms of SARS within 24 hours of her hospitalisation.

A similar policy was adopted by other hospitals and would cause a shortage of hospital beds and strain EDs in the weeks to come.

Despite being a SARS hospital, we were admitting between 5 to 15 nonSARS patients a day. These were TTSH patients who were too ill to be transferred to other hospitals.

\section{WEEK 6 (18-24 APRIL 2003)}

We were one of the first to suspect that SARS had spread to the community when a family of eight were referred by a general practitioner for fever. The grandmother of the family had just died from bronchopneumonia. There was no obvious contact history. We admitted all eight of them. They were the herald of the next cluster. Contact history was important but was no longer necessary in suspecting SARS in a patient. Previous to this there had been tenuous links to healthcare institutions like a taxi driver who had ferried passengers 
to hospitals, visitors to hospitals or staff of hospitals.

We were later to discover that the grandfather had contracted SARS at the biggest wholesale vegetable centre in the country where a SARS victim had worked for several days before seeking medical attention. The government closed the centre for 14 days.

With the spread of SARS into the community, we now categorised the patients we screened into low, moderate or high risks instead of no, low, moderate, or high.

The definition became:

- Low risk-no definite exposure with or without symptoms

- Moderate risk-contact or travel with or without symptoms

- High risk-contact or travel history with a temperature of $38^{\circ} \mathrm{C}$ or more

\section{WEEK 7 (25 APRIL-1 MAY)}

We reorganised and increased the tentage area as the number of patients with low risk increased. (Figure 1 shows the plan for the work area for SARS screening.)

The biggest problem now was the weather, which averaged $34^{\circ} \mathrm{C}$. The staff working in the frontline were drenched within minutes of stepping out into the working area. They were instructed to drink water and isotonic fluids before starting their shifts and a "water" break after working 90 minutes was strictly enforced. Two staff were assigned to keep track and despite this, two staff did suffer giddy spells but fortunately recovered very quickly.

WEEK 8, 9, 10 (2 MAY-29 MAY)

There were high hopes that Singapore would be SARS free on the 18 May 2003 but a patient we had admitted on the 11 May 2003 (for whom to date we are unable to obtain a contact history) turned for the worse and tested positive for SARS. Two previous tests for coronavirus had been negative.

\section{WEEK 11}

Singapore celebrated as the country was taken off WHO list of SARS affected countries. $^{7}$ TTSH ED prepares for resumption of its normal functions.

\section{LESSONS LEARNT}

\section{Infection control measures}

It is the nature of epidemics to be unpredictable. ${ }^{8}$ This was made worse because SARS was a new disease. In the early days of the outbreak, we were uncertain of which level of infection control measures to adopt and there was a concern about causing unwarranted public panic as well.
It took us five days before we set up a screening centre at the entrance of the ED and a few more days before all staff involved in patient care wore full PPE.

We were fortunate that the institution had in place mask fitting as part of its orientation programme. However, many of the ED staff still had to undergo mask fitting and a check four weeks into the crisis found that a few had not done so. This is crucial for staff protection. Staff must wear the correct size N95 facemask.

Audit is very important. It should be recorded if staff have undergone mask fitting, training in putting on gown and removing them, and in infection control measures such as changing gloves and wiping down their stethoscopes between patients. We assigned senior staff to be our main auditor. This role was very important in enabling us to maintain strict compliance of infection control measures.

We were fortunate to have enough outdoor space to separate the different categories of patients. This we believe was one of the reasons why we did not have any transmission of the disease within the department.

\section{Communication}

This was one of the most critical components in our management of the SARS crisis.

At the hospital level, the ED head was kept updated of the latest developments at the daily meetings chaired by the chief executive officer. The ED's input was taken into consideration during decision making and there was direct access to senior management and this was of importance in obtaining logistical support and in resource management.

The relationship between ED and ID physicians has always been close and the two groups had worked together previously during the Nipah virus out-

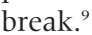

This was why ID physicians were comfortable visiting the ED and sharing any new information that they had available as well as exchanging ideas with the ED staff.

The ED also obtained up to date information especially of at risk areas from the epidemiology team. It also helped that one of them had worked as a medical officer in the ED previously and was familiar with our work processes.

These open channels between the ID, epidemiology, and ED teams allowed for the early identification of new cases and clusters like the family of eight and those from other hospitals. Early identification allowed for contact tracing and isolation to be achieved much faster.
This open channel of communication was also present between the staff who ran the ED hotline and the home surveillance team. There were a few SARS patients who had been discharged initially but were subsequently recalled and admitted because of this close working relationship between the two teams.

At the department level, the open letter issued by the HOD was one form of communication. It is not easy to disseminate ever changing information in an ED as most staff work shifts. Briefings and debriefings by senior staff both medical and nursing became one of the most important lines of communication for the department.

\section{Command and control}

In a crisis, the chain of command must be clear to all concern. Fortunately, in ED TTSH the medical, nursing, and support staff have the same head of department. This made it easier for decisions to be made and carried out as the final responsibility belonged to only one person, the head of department. This made it easier for the staff to respond to changes.

Staff were frequently reminded in the first few weeks to follow only instructions from the head of department. The more senior the staff, the harder it was for them to comply and there was a tendency for this group to issue instructions without clearing with the head of department. This can cause confusion to the ground. It is important for the head of department to maintain discipline in such situations.

\section{Staff morale}

The fear felt by the staff must be acknowledged. We admitted our ignorance and were open about any information we received. We explained the rationale behind decisions and changes that were made.

The staff were prepared from the start that the SARS crisis would be a marathon and not a sprint. They were warned to expect many changes within a day and that decisions may be reversed frequently depending on whatever information we receive. It was very important to the staff for the head of department and senior staff to be visible in the ED during this period.

The hospital management was very supportive in providing funds for us to provide meals and recreational activities for the staff. Public support was also a great morale booster.

However, the staff did face prejudices. "They were fighting the unknown virus as well as fighting discrimination."10 


\section{Patient behaviour}

Contact with a known case is important but this may not be evident at the initial assessment. ${ }^{11}$ In the case of the family of eight, they did not know but in at least one case, the patient was not forthcoming with the truth. This behaviour is not peculiar to Singapore. It also occurred in Taiwan (L M Wang, personal communication).

We postulate that these patients were in denial.

Fortunately, we learnt this lesson very early during the crisis. We decided to actively search the computerised database of SARS patients and contact for every patient we screened even though this added to the turnaround time at screening.

\section{Community spread}

We were expecting the worse from the start of the crisis. We suspected that it would be a matter of time before SARS spread to the community. Even though contact history was an important discriminator $^{11}$ for us, we did not rely on it too strongly. We admitted all patients who fitted the clinical picture of SARS ${ }^{12}$ even if they did not have a contact history. To date, we have yet to trace the contact history of the last patient in Singapore diagnosed to have probable SARS.

\section{Clinical expertise}

We had 24 hour senior doctor cover. This ensured that the quality of care did not suffer during the crisis. It also allowed us to gain clinical experience of this new disease very quickly. We discovered like Hong Kong that although fever is a cardinal symptom, ${ }^{11}$ it could be absent in a minority of patients.

The presence of this leadership was very important in giving the staff confidence as they handled multiple changes and new problems.

\section{Conclusion}

We have survived the SARS crisis by expecting the worse and hoping for the best. We are now moving from crisis to a transition period.

\section{ACKNOWLEDGEMENTS}

I would like to thank the staff of ED, TTSH for their support during this SARS crisis. They are a great team to work with.

Emerg Med J 2003;20:501-504

\section{Author's affiliations}

E Seow, Tan Tock Seng Hospital, Emergency Department, 11 Jalan Tan Tock Seng,

Singapore, 308411; eillyne_seow@ttsh.com.sg

\section{REFERENCES}

1 Goh LG. Reflections on the Singapore SARS outbreak, commentary. SMA News 2003;35:5.

2 The Straits Times. http:// straitstimes.asial .com.sg/columnist/0,1886,56178860,00.html.

3 Leo YS, Chen M, Heng BH, et al. Severe acute respiratory syndrome-Singapore 2003. MMWR 2003;52:405-11.

4 Ministry of Health, Singapore. Enhanced precautionary measures to break SARS transmission, 22 March 2003. http:// www.asial.com/straitstimes/

5 Reference withdrawn.

6 Chee YC. SARS (and me) (Part 1). SMA News March 2003:35:6.

7 WHO. http://www.who.int/csr/don/ 2003_05_30a/en/.

8 Bloom BR. Lessons from SARS. Science 2003;300:701.

9 Tambyah PA. The Nipah virus outbreak - a reminder. Singapore Med $J$ 1999;40:329-30.

10 Chee YC. Heroes and heroines of the war on SARS. Singapore Med J 44:221-8.

11 Tomlinson B, Cockram C. SARS: experience at Prince of Wales Hospital, HongKong. Lancet 2003;361:1486-7.

12 Hsu LY, Lee CC, Green JA, et al. Severe acute respiratory syndrome (SARS) in Singapore: clinical features of index patient and initial contacts. CDC 2003;9:1-7. 